\title{
Three-Dimensional Statistical Analysis of Sulcal Variability in the Human Brain
}

\author{
Paul M. Thompson, Craig Schwartz, Robert T. Lin, Aelia A. Khan, and Arthur W. Toga \\ Laboratory of Neuro Imaging, Department of Neurology, Division of Brain Mapping, UCLA School of Medicine, Los \\ Angeles, California 90095
}

Morphometric variance of the human brain is qualitatively observable in surface features of the cortex. Statistical analysis of sulcal geometry will facilitate multisubject atlasing, neurosurgical studies, and multimodality brain mapping applications. This investigation describes the variability in location and geometry of five sulci surveyed in each hemisphere of six postmortem human brains placed within the Talairach stereotaxic grid. The sulci were modeled as complex internal surfaces in the brain. Heterogeneous profiles of three-dimensional (3D) variation were quantified locally within individual sulci.

Whole human heads, sectioned at $50 \mu \mathrm{m}$, were digitally photographed and high-resolution 3D data volumes were reconstructed. The parieto-occipital sulcus, the anterior and posterior rami of the calcarine sulcus, the cingulate and marginal sulci, and the supracallosal sulcus were delineated manually on sagittally resampled sections. Sulcal outlines were reparameterized for surface comparisons. Statistics of 3D variation for arbitrary points on each surface were calculated locally from the standardized individual data. Additional measures of sur-

Modern whole brain imaging and histological techniques have allowed the neuroscience community to gather a detailed inventory of information on the anatomical structure of individual brains. In vivo imaging techniques, such as positron emission tomography (PET) and functional magnetic resonance imaging (MRI), have also made it possible to map functional areas of the human brain with respect to its anatomy. However, striking variations exist across individuals in the internal and external geometry of the brain (Sanides, 1962). Such normal variations in the size, orientation, topology, and geometric complexity of cortical and subcortical structures have complicated the goals of developing standardized representations of human neuroanatomy and of comparing functional and anatomic data from many subjects.

The quantitative comparison of brain architecture across different subjects requires a common coordinate system to express

Received Dec. 18, 1995; revised March 19, 1996; accepted April 5, 1996.

This work was generously supported by a Fulbright Scholarship from the U.S.U.K. Fulbright Commission, London, by Grant G-1-00001 of the United States Information Agency, Washington, D.C., and by a predoctoral fellowship of the Howard Hughes Medical Institute (P.M.T.). Additional support was provided by the National Science Foundation (BIR 93-22434), the National Library of Medicine (LM/MH05639), the NCRR (RR05956), and the Human Brain Project, which is funded jointly by the National Institute of Mental Health and the National Institute on Drug Abuse (P20 MH/DA52176). Special thanks go to the anonymous reviewers for their helpful comments, and to Andrew Lee and Lynn Hodges for their assistance in preparing the figures for this paper.

Correspondence should be addressed to Dr. Arthur W. Toga, Reed Neurological Research Center, Room 4238, Laboratory of Neuro Imaging, 710 Westwood Plaza, Los Angeles, CA 90095-1769.

Copyright (C) 1996 Society for Neuroscience $0270-6474 / 96 / 164261-14 \$ 05.00 / 0$ face area, extent in three dimensions, surface curvature, and fractal dimension were used to characterize variations in sulcal geometry.

Paralimbic sulci exhibited a greater degree of anteriorposterior variability than vertical variability. Occipital sulci displayed the reverse trend. Both trends were consistent with developmental growth patterns. Points on the occipital sulci displayed a profile of variability highly correlated with their 3D distance from the posterior commissure. Surface curvature was greater for the arched paralimbic sulci than for those bounding occipital gyri in each hemisphere. On the other hand, fractal dimension measures were remarkably similar for all sulci examined, and no significant hemispheric asymmetries were found for any of the selected spatial and geometric parameters. Implications of cortical morphometric variability for multisubject comparisons and brain mapping applications are discussed.

Key words: brain mapping; cortex; stereotaxic methods; sulcus; 3D image reconstruction; morphometry

the spatial variability of features from different individuals (Evans et al., 1996). Stereotaxic localization, for example, provides a quantitative system of reference in human functional studies and stereotaxic surgical procedures. The usefulness of these atlas systems depends on how closely the brains of individual subjects match the representation of anatomy in the atlas. Anatomic correspondence is especially critical at functional interfaces and cytoarchitectonic boundaries, such as deep internal banks of primary sulci (Rademacher et al., 1993). The inherent neuroanatomic variability between individuals, as well as the numerous differences between stereotaxic systems themselves (Burzaco, 1985), warrants the development of a well defined reference system able to represent and classify idiosyncratic, age-related, developmental, or pathological variations in anatomy.

\section{Sulcal anatomy}

Sulci were chosen as a basis for structural analysis of the internal surface anatomy of the brain because they separate functionally distinct regions of the brain and provide a natural topographic partition of its anatomy. Whereas functional or architectonic boundaries are not directly visible with MRI, these boundaries bear a well documented and characteristic relation to the banks, depths, secondary branches, and internal points of confluence of the sulci (Watson et al., 1993; Roland and Zilles, 1994). Moreover, most of the junctional zones between adjacent microanatomic fields run along the beds of major or minor cortical sulci (Sanides, 1962). 
Despite their anatomic and functional significance, even the gyri and sulci that consistently appear in all normal subjects exhibit pronounced variability in size and configuration (Bailey and von Bonin, 1951; Ono et al., 1990). Striking intersubject variations in sulcal geometry have been reported in primary motor, somatosensory and auditory cortex (Rademacher et al., 1993), primary and association visual cortex (Stensaas et al., 1974), frontal and prefrontal areas (Rajkowska and Goldman-Rakic, 1995), and lateral perisylvian cortex (Steinmetz et al., 1990). Ultimately, direct reference to the internal sulcal surfaces that frame architectonic fields may present a more reliable basis for functional mapping than reference to a single standard or idealized brain (cf. Steinmetz et al., 1990; Rademacher et al., 1993).

Although the intrinsic variability in sulcal configuration across individuals is well known, the ranges of these normal variations have not yet been determined. Previous sulcal variability studies have been based on pneumoencephalograms (Talairach et al., 1967), series of 5-mm-thick or 9-mm-thick magnetic resonance (MR) images (Missir et al., 1989; Steinmetz et al., 1989, 1990), and MR-derived surface renderings of the cortex (Vannier et al., 1991). These investigations have yielded much useful qualitative information on the positional variability of the major sulci. However, they represented sulci as superficial curves between the outer extremities of opposing gyri rather than as complex threedimensional (3D) architectonic surfaces, which merge and branch deep inside the brain.

Precise quantitative information on sulcal variability has been limited by image resolution and sampling frequency. Because of the large spacing between sections, it has not always been possible to trace the course of individual sulci from one anatomic section to the next (Missir et al., 1989). Serial sectioning and full-color digital photography of whole human heads offers the highest resolution technique for imaging sulcal anatomy (Toga et al., 1995). Compared with the $128^{2}-512^{2}$ pixel gray-scale images of 1.5-2.0-mm-thick contiguous sections provided by MRI (Steinmetz et al., 1990; Vannier et al., 1991), modern whole-brain physical sectioning (cryosection) procedures regularly generate $1024^{2}-2048^{2}$ pixel resolution, full-color images of contiguous sections 20-50 $\mu \mathrm{m}$ thick (Quinn et al., 1993; Toga et al., 1994a,b, 1995; Thompson et al., 1996). In addition, these data provide excellent color pigment differentiation and texture contrast at the cortical laminae and at gray-white matter interfaces flanking the sulci. Accordingly, high-resolution images of cryosectioned human anatomy offer the spatial and densitometric resolution necessary for accurate quantitative analysis of the internal surface anatomy of the brain.

To spatially characterize the morphometric variability in the interior surface geometry of the brain, we modeled the major sulci in $3 \mathrm{D}$. Their internal surfaces were modeled using a multiresolution parametric mesh approach. In addition, we describe a mathematical framework for examining sulcal variability in three dimensions.

The following primary sulci were selected for 3D reconstruction and analysis: the supracallosal sulcus, the cingulate and marginal sulci, the anterior and posterior rami of the calcarine sulcus, and the parieto-occipital sulcus. As major functional interfaces in the brain, these primary sulci are easily identifiable, mark critical gyral and lobar boundaries, and penetrate sufficiently deeply into the brain to introduce a topological decomposition of its volume architecture. Consequently, their internal trajectories are sufficiently extended inside the brain to reflect subtle and distributed variations in neuroanatomy between individuals.

\section{MATERIALS AND METHODS}

Cryosectioning and image acquisition. The protocol for whole human head cryosectioning and digital image capture was performed as described previously (Quinn et al., 1993; Toga et al., 1994a,b, 1995). Six normal cadavers (aged 72-91 years, 3 males) were obtained optimally within 5-10 hr postmortem through the Willed Body Program at the UCLA School of Medicine. Exclusion criteria were applied to ensure that, in each case, the primary cause of death had not involved any pathological or traumatic impact on the brain. (The primary causes of death were recorded as follows: pulmonary edema/congestive failure; heart failure; cirrhosis; bacterial pneumonia; respiratory failure; and malignant melanoma. All experimental procedures were conducted in accordance with UCLA Medical Center policies on donor confidentiality and Federal Health and Safety Codes.)

Specimens were prepared for sectioning in three of the six cases by perfusing with $8 \%$ formalin, cryoprotecting with $10 \%$ glycerol, freezing in isopentane and dry ice, and blocking in green tempura paint and $3 \%$ sucrose solution. The heads were cryosectioned at $-25^{\circ} \mathrm{C}$ through the horizontal plane in $50 \mu \mathrm{m}$ increments on a heavy-duty cryomacrotome (PMV, Stockholm, Sweden). In each case, the cranium was left intact to preserve the brain's in situ conformation and to prevent relaxation of the cerebellum and splaying of the interhemispheric vault (Toga et al., 1994b). The cryomacrotome was equipped with a high-resolution Digistat $1024^{2} \times$ 24-bit full-color camera (Dage-MTI, Michigan City, IN) for digital image capture of the cryoplaned specimen. Spatial integrity of the data volume was guaranteed by digitizing the 1200-1300 serial images from the specimen blockface itself during the sectioning process (Quinn et al., 1993). This protocol ensures that each consecutive section is in perfect register with its predecessors. In the three remaining cases, the whole nonperfused head was immediately removed and placed on ice and saline, and extraneous soft tissues were removed from the skull. The specimen was frozen in situ in isopentane chilled by an external bath of liquid nitrogen. The occipital region of the frozen calvarium was removed with a Stryker bone saw, and the specimen was blocked into freezing distilled water before cryosectioning.

$3 D$ image reconstruction and transformation to stereotaxic space. Image data from each of the six heads were assigned real-world coordinate values in micrometers for width, height, and depth. 3D reconstruction of the serial images resulted in a digital data volume that was subsequently transformed into the Talairach stereotaxic coordinate system (Talairach et al., 1967).

A series of steps were required to map each 3D data volume into Talairach space, using the transformations specified in the atlas (Talairach et al., 1967). The locations of the superior margin of the anterior commissure and inferior margin of the posterior commissure were identified and described in pixel coordinates. Piecewise affine transformations were used to vertically align the interhemispheric fissure and transpose the volume to a horizontal origin at the anterior commissure-posterior commissure (AC-PC) line. As prescribed by the Talairach system, different amounts of scaling were then imposed on 12 rectangular regions of the brain, defined by vectors from the AC-PC line to the extrema of the cortex. A complete set of images was then generated for all six heads by digitally resampling the volume at $500 \mu \mathrm{m}$ increments in each of the sagittal, coronal, and horizontal planes.

Criteria for delineating sulci. After placement of the standardized individual data into the Talairach stereotaxic grid, the complex internal paths of the major deep sulcal fissures in the brain were reconstructed using a contour-based system. With the aid of an interactive contouring program developed in our laboratory, all sulci were outlined manually according to the detailed anatomic criteria set out in Steinmetz et al. (1989). Additional formal guidelines were devised and applied when identifying the exact course of individual sulci in three dimensions (see Fig. 1).

Cellular interfaces between gray and white matter were used to define the opposing banks of the sulci, rather than the more diffuse boundary of gray matter at the external limit of the cortical layer. The high densitometric gradient of the anatomic images at these banks allows them to be identified with single-pixel accuracy. Consequently, the internal path of each sulcus was defined as the medial curve equidistant between the opposing white matter banks on either side. In rare cases, in which the white matter was faint, adjacent sections were viewed for additional information. At high magnification, the outline of each sulcus was defined to be the medial axis equidistant from each bank. This contour was traced manually in all the sagittal sections in which it could be distinguished. At the external cerebral surface, the convex hull of the cortex served as an exterior limit. 
A

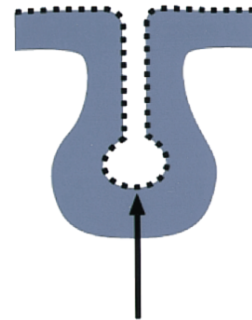

CSF/Gray matter interface
B

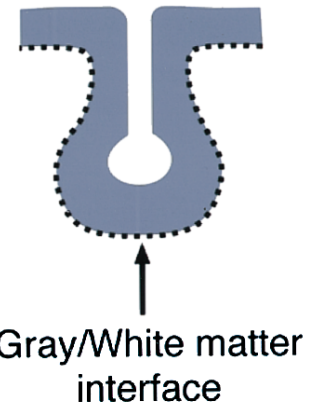

C

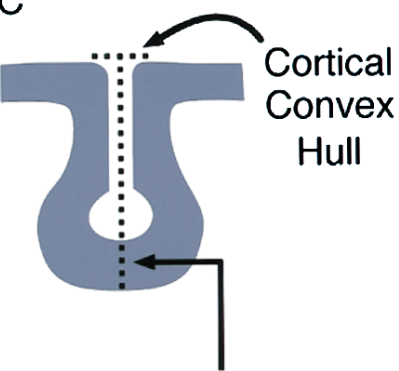

\section{Medial Axis between Gray/White interfaces}

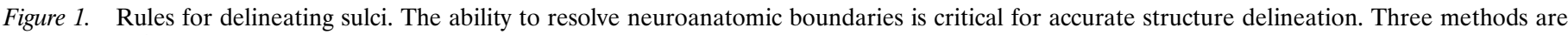

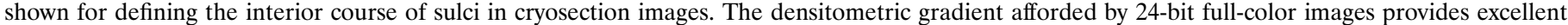

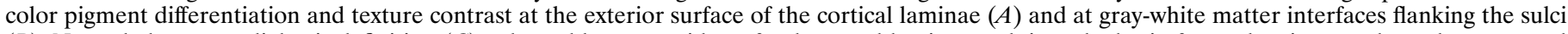

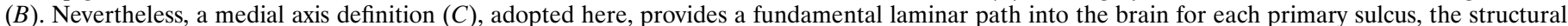

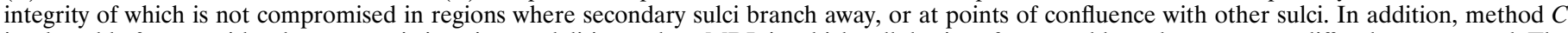

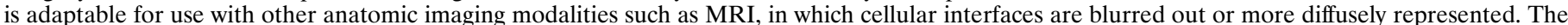

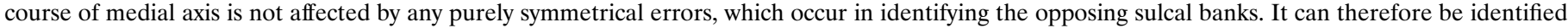
in an accurate and reproducible way, even in low-contrast imaging modalities.

Sulcal outlines were digitized as a cursor was moved over a highly magnified image of each slice along the curvilinear path of each sulcus. As a guide to the anatomic relations of the selected sulci, Figure 2 shows a sagittal projection of all the contours traced in the left hemisphere of one specimen. The stereotaxic locations of contour points were derived from the data volume, and all 60 stacks of sulcal contours were stored in files as numerical coordinate values, before 3D surface analysis.

Surface reconstruction from planar cross-sections. Interactive outlining of sulci, as described above, resulted in a sampling of $\sim 15,000$ points per sulcus. Although this dense system of points captures the details of each sulcal surface at a very local level, their spatial distribution is not quite uniform and is arbitrarily dependent on how the sagittal sampling planes intersect the sulcus being outlined. To eliminate this dependency, a program was developed that used the digitized outlines of the sulci as the basis for deriving a standard surface representation of the same type for each sulcus. For each sulcus outlined, the algorithm generates a parametric grid of $100 \times 150$ uniformly spaced points that act as nodes in a regular rectangular mesh stretched over the sulcal surface (Fig. 3). Full

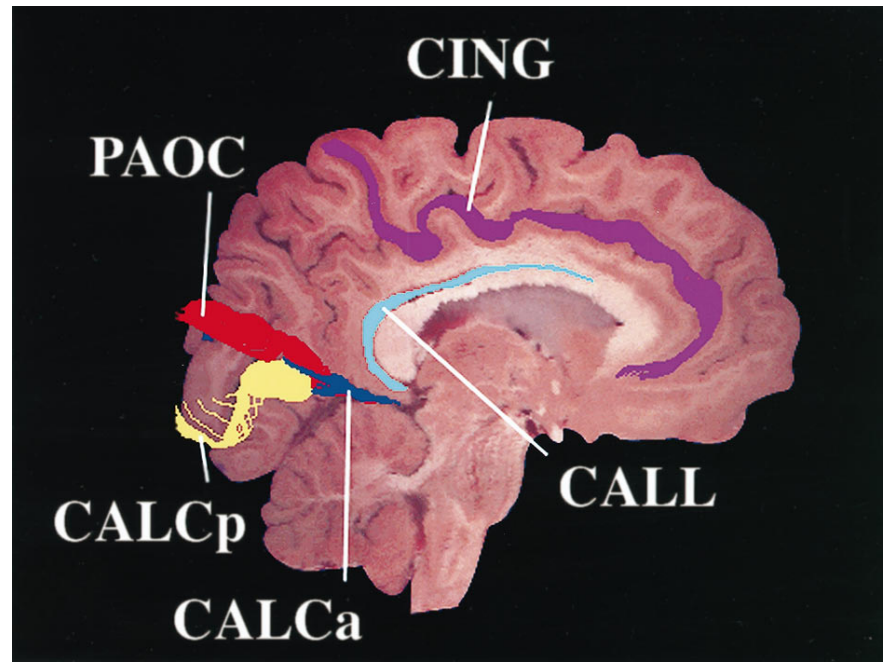

Figure 2. Sagittal projection of the full set of sulcal contours traced in the left hemisphere of a single brain. These sets of contours were derived from the full series of sectional images spanning the left hemisphere of one brain specimen. Orthogonally projected contours of the anterior and posterior rami of the calcarine sulcus $(C A L C a$ and $C A L C p)$, as well as the cingulate $(C I N G)$, supracallosal $(C A L L)$, and parieto-occipital $(P A O C)$ sulci, are shown overlaid on one representative sagittal section.

technical details of the mesh construction algorithm can be found in Thompson et al. (1996). Each resultant surface mesh is analogous in form to a regular rectangular grid, drawn on a rubber sheet, which is subsequently stretched to match all the data points. This scheme provides a means for converting dense systems of points, sampled during outlining, into fully parametric surfaces that can be analyzed, visualized, and com-

\section{PARAMETRIC MESH CONSTRUCTION}

$$
\text { Regular 2D Grid }
$$

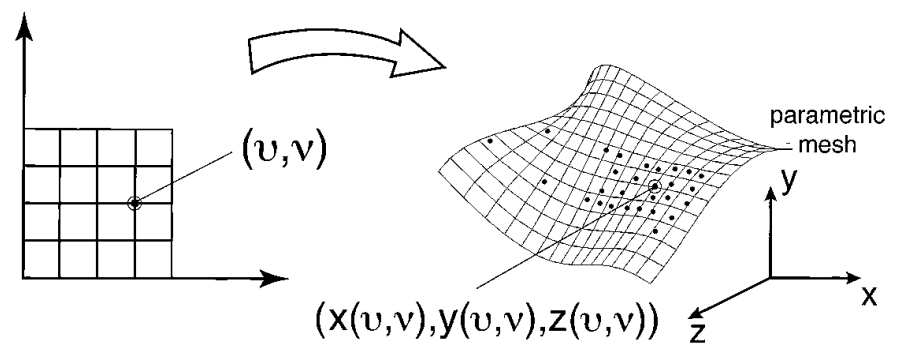

...... points sampled during outlining of structures in serial cross-sections

Figure 3. Parametric mesh construction. The outlining process generates a densely sampled set of points, which are known to be located on the internal surface of a sulcus (indicated by isolated points, above right). These points, however, are not distributed uniformly on the sulcal surface. Isolation of points that correspond geometrically involves the molding of a lattice-like mesh onto the geometric profile of the surface so that each point on the mesh can be averaged with its counterparts on other surfaces. The concept is similar to that of a regular net being stretched over an object. Under certain strict conditions, the imposition of regular grids onto biological surfaces permits cross-subject comparisons by specifying a computed correspondence along the outline arcs and within the interior of the structures (Bookstein, 1985). The imposition of an identical regular structure on surfaces from different specimens allows surface statistics to be derived. Local statistical comparisons are then made by associating points with identical grid locations within their respective surfaces. One condition that must hold for the comparisons to be valid is that landmark points and curves known to the anatomist appear in corresponding locations in each parametric grid. The appendix describes a battery of tests that were performed to confirm that this condition was satisfied. Mesh partitioning strategies (see Appendix) were also used to ensure the accuracy of the computed correspondences at complex anatomic boundaries. 
pared geometrically and statistically. Under certain strict conditions, the imposition of regular grids onto 3D biological surfaces permits crosssubject comparisons by specifying a computed correspondence along the outline arcs and within the interior of the structures (Bookstein et al., 1985). For the comparisons to be valid, anatomically defined landmark points and curves must appear in corresponding locations in each parametric grid. A battery of tests were conducted to confirm that this condition was satisfied. These tests are described in the Appendix. Mesh partitioning strategies (see Appendix) were also used to ensure that the computed geometric correspondences were accurate at complex anatomic junctions and boundaries.

Measures of spatial extent, surface curvature, area and fractal dimension. Parameterization of the sulcal outlines from the six specimens resulted in a set of six regular parametric meshes of identical resolution for each sulcus in each hemisphere. Because the underlying parametric grids are regular and have the same nodal structure, these mesh-based models permit comparison of several models of the same sulcus (Bookstein et al., 1985) and enable computation of local statistical measures and geometric parameters, such as surface area, curvature indices, and fractal dimension

The anteroposterior, vertical, and lateral extents of all 60 sulci were determined from the digitized outlines. Surface area measures were also calculated. In addition, because one of the most prominent features of the human cerebral cortex is its high degree of convolution, normalized curvature measures were computed for all 60 sulcal surfaces. The mathematical form of the curvature measure is explained in the Appendix. Both surface area and curvature measures were defined on the parametric meshes instead of the sample points initially acquired for each sulcus. One advantage of this approach is that the spatial resolution of the meshes is standardized, allowing the geometric measures in each case to be independent of the sampling frequency at which the contours were originally digitized. Finally, the fractal dimension of each sulcal surface was calculated.

Computation of displacement maps on sulcal surface meshes. In our formulation, all 60 meshes representing sulcal surfaces were defined on a grid of the same resolution $(100 \times 150)$ so that the relationship between two sulci of the same type could be represented as a map that displaces one surface mesh onto another in stereotaxic space. For each and every point on a surface mesh $\mathbf{M}_{1}$, and every point on a similar mesh $\mathrm{M}_{2}$, the two points were matched if they had the same grid location within their respective surfaces. For each such association, the discrepancy was computed as a 3D displacement vector between corresponding nodal points. Ultimately, this procedure yielded a full displacement map for every pair of surfaces of the same type.

Furthermore, an average surface representation was derived for each sulcal type by averaging the $3 \mathrm{D}$ position vectors of nodes that correspond, across all six specimens (Fig. 4). This representation also provided a means for quantifying the local variability of internal points in a sulcal surface based on our sample of parametric surfaces taken from our six specimens. Local measures of spatial variance are based on the availability of an average surface representation together with the concept of a sulcal mapping, which is a type of displacement map (Fig. 5). A complete mathematical formulation of this notion can be found in Thompson et al. (1996), where similar maps are used to derive a high-dimensional probability measure for detecting abnormalities in the anatomy of new subjects. Briefly, a sulcal mapping is specified by a set of 3D displacement vectors that take each nodal point from its latticial position in the average surface mesh onto its corresponding point in a mesh representing the same surface in another brain (Martin et al., 1994; Sclaroff and Pentland, 1994).

Sulcal maps were calculated for all 60 surfaces, relating each one to its respective average surface (Fig. 5). The profile of variability across each surface was then derived locally from the sulcal maps as an SD for each internal point in Talairach millimeters. The appropriate numerical value was obtained at each grid point as the root mean square (rms) magnitude of the 3D displacement vectors assigned to that point in the six surface maps from average to specimen. Finally, the range of this variability parameter was mapped, via a linear look-up table, onto a standard color range. Local profiles of variability were visualized (using Data Explorer 2.1, IBM Visualization Software) by adding a range of colors to the surface representation of each sulcus. All 3D reconstruction programs were written in $\mathrm{C}$ and executed on DEC $\alpha$ AXP3000 work stations running OSF-1.

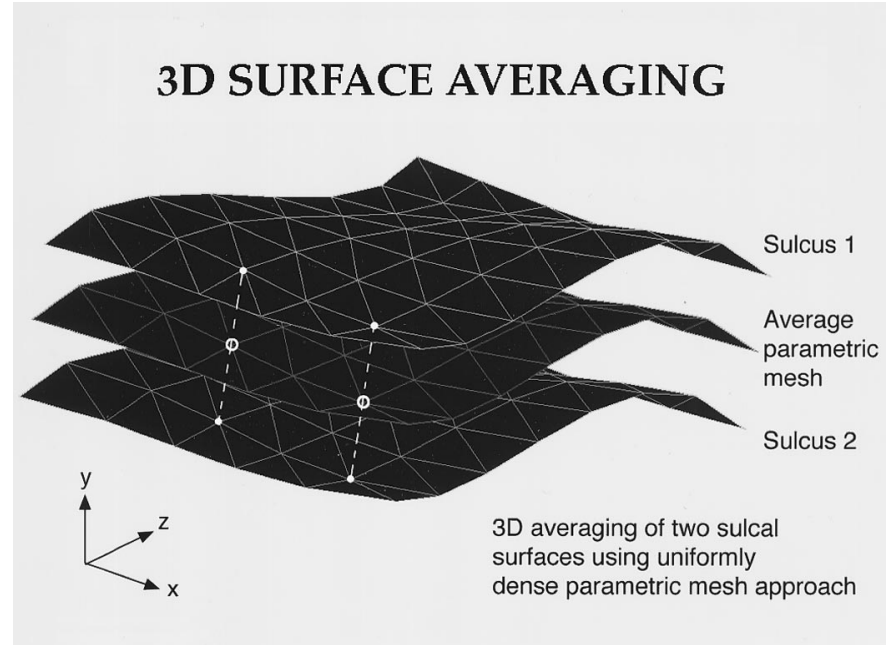

Figure 4. 3D surface averaging. To determine the discrepancy between two surfaces in the same stereotaxic system, a mesh construction algorithm generates a structured pattern of sample points at corresponding positions on surfaces outlined in different specimens, before examining the distances between the sets of corresponding points (Sclaroff and Pentland, 1994). Because the resolution of the meshes is standardized, the averaging of the $3 \mathrm{D}$ position vectors of corresponding nodes on meshes from each specimen yields an average surface representation for each sulcus.

\section{RESULTS}

From the broad spectrum of geometric and statistical variables examined here, two general principles are evident. First, striking directional trends were observed (Figs. $6 A, B, 7$ ) when the profiles of $3 \mathrm{D}$ spatial variability were broken down into components along each orthogonal dimension of stereotaxic space. The direction of greatest variability was consistently found to be vertical for the occipital sulci and anterior-posterior for the paralimbic sulci in both hemispheres. Second, indices that reflect the overall magnitude of variability for a sulcal surface were remarkably consistent from one sulcus to another (Fig. 6A). However, these global measures tended to obscure the distinctly heterogeneous profiles of variability across the surfaces of individual sulci (see Fig. 8). In particular, local variability was consistently higher toward the exterior cortical surface (see Fig. $8 B$ ).

For the parieto-occipital, posterior calcarine, and cingulate sulci, the associated confidence limits on 3D variation increased from an SD of 8-10 mm internally to a peak of $17-19 \mathrm{~mm}$ at the exterior cerebral surface. This phenomenon is not surprising given that the Talairach system fixes the locations of the two commissures and is accordingly more effective at reconciling population variances in structures close to these control points (Steinmetz et al., 1989).

Intersubject variations for the full set of selected geometric and statistical measures are illustrated in Figures 6-9. The 3D variation zones of the 60 sulcal surfaces were in agreement with previous two-dimensional (2D) studies based on sagittal MR images and pneumoencephalograms (Talairach et al., 1967; Missir et al., 1989; Steinmetz et al., 1989, 1990; Vannier et al., 1991). Lateral variation zones were not addressed in previous studies and are shown in Figure $9 C$. As was also expected for the structures examined, no significant hemispheric asymmetries were observed for any of the selected geometric parameters. 


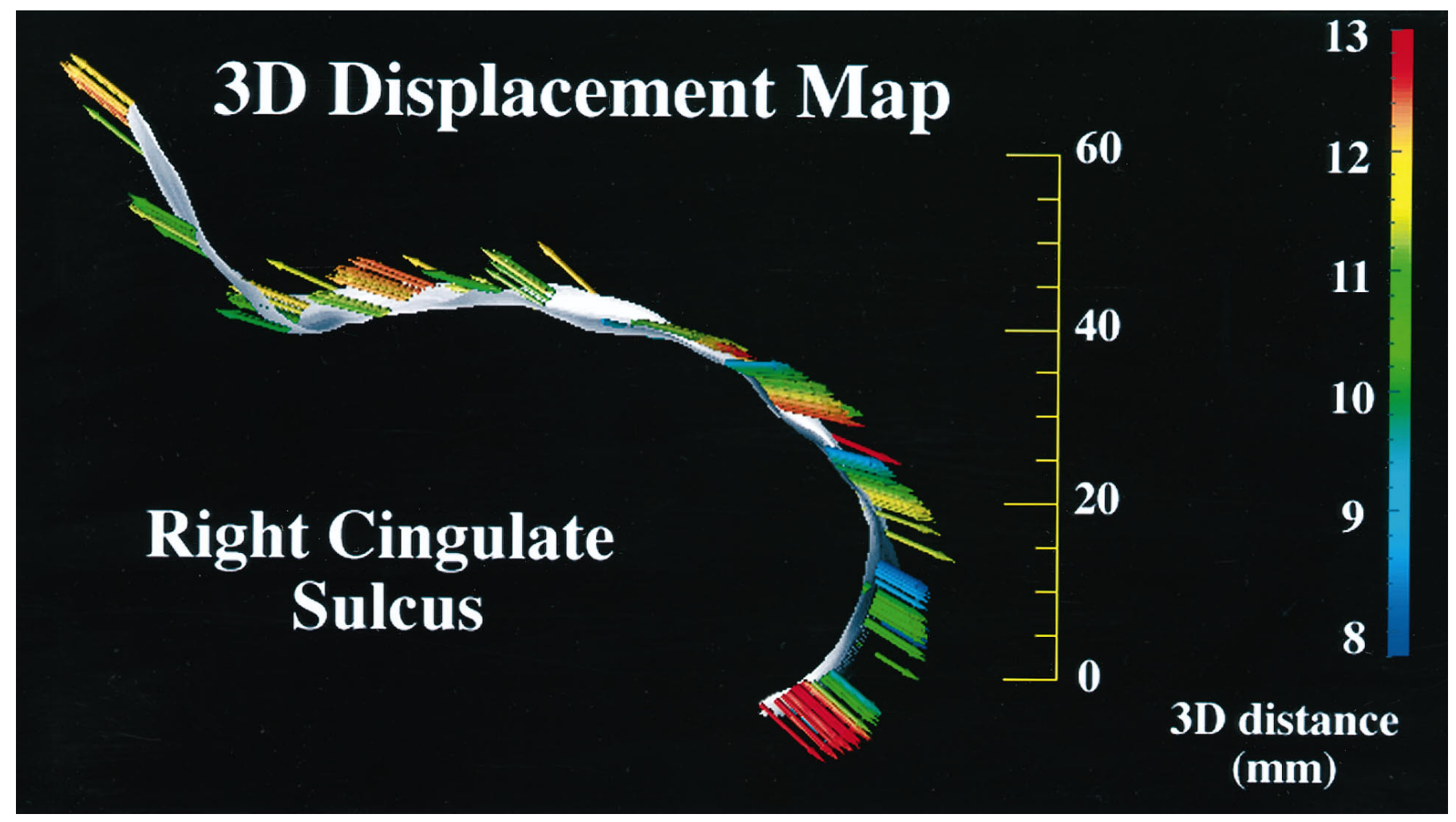

Figure 5. A 3D displacement map shown on a 3D representation of the average right cingulate sulcus. Local discrepancies between individual sulci and their respective average surface can readily be calculated. Both the magnitude and direction of such surface discrepancies are indicated by arrows that originate at points defined by the mesh. The map shown displaces the average representation of the right cingulate sulcus onto the equivalent surface in a randomly selected specimen brain. Notice that the mesh in this figure contains a reduced number of points for the convenience of illustration. The coronal plane through the anterior commissure $(y=0)$ divides the anatomical architecture into two regions, which are subjected, by the Talairach transform, to different scaling transformations in the anterior-posterior direction. This aspect of the stereotaxic transform may explain why the directional bias of local anatomic variation differs considerably for sulcal points on each side of this coronal plane.

\section{Directional trends}

Clear directional trends were revealed when the measures of 3D spatial variability for each sulcus were decomposed into components along each of the three orthogonal axes of stereotaxic space. The variability in position exhibited by each sulcus was not isotropic, although its inherent directionality was different for different classes of sulci. All six occipital sulci displayed greatest variability in the vertical direction, whereas all four paralimbic sulci exhibited maximal variability in the anterior-posterior direction. For example, confidence limits on spatial variation for the posterior calcarine sulcus were considerably wider in the vertical dimension than in the anterior-posterior dimension (vertical rms deviations: $10.78 \pm 2.23 \mathrm{~mm}$, left hemisphere; $12.08 \pm 2.56 \mathrm{~mm}$, right hemisphere; anterior-posterior rms deviations: $3.96 \pm 1.65$ $\mathrm{mm}$, left hemisphere; $2.98 \pm 1.29 \mathrm{~mm}$, right hemisphere). By contrast, for the cingulate sulcus, confidence limits spanned a smaller range in the vertical dimension than in the anteriorposterior dimension (vertical rms deviations: $5.38 \pm 2.58 \mathrm{~mm}$, left hemisphere; $4.70 \pm 1.91 \mathrm{~mm}$, right hemisphere; anteriorposterior rms deviations: $9.54 \pm 4.53 \mathrm{~mm}$, left hemisphere; $9.67 \pm$ $2.12 \mathrm{~mm}$, right hemisphere). Of the three directional components, the lateral component of spatial variability was consistently lowest of all. This was the case in both hemispheres for every sulcus examined (mean lateral $\mathrm{rms}$ deviation between subjects $=2.42$ $\mathrm{mm}$, all sulci).

When the magnitude of the local variability was analyzed for each sulcus, further complex trends were observed, in addition to those relating to the direction of maximal variation. Multiple regression analysis confirmed that the magnitude of local variability for points on the occipital sulci (PAOC, CALCa, CALCp) is strongly dependent on their radial distance from the posterior commissure. Points on the parieto-occipital sulcus, for example, displayed a profile of variability (defined as the 3D rms distance for each grid point in the mesh from the average PAOC) highly correlated with their 3D distance from the posterior commissure (correlation coefficient: $r=0.85$; coefficient of determination: $r^{2}$ $=0.715)$. The rise in the variability measure toward the exterior cerebral surface is illustrated in Figure $7 B$. Analysis of a densely sampled series of sagittal sections of the average PAOC in both hemispheres revealed that the $3 \mathrm{D}$ rms variability measure was, in each case, independent of lateral position in the brain $\left(r^{2}=0.001\right.$, left hemisphere; $r^{2}=0.004$, right hemisphere).

For the sulcal surfaces shown in Figure $8 B$, the measure of $3 \mathrm{D}$ rms variability rose at an estimated rate of $0.084 \pm 0.005 \mathrm{~mm}$ per millimeter distance from the posterior commissure (PC) in the anterior ramus of the right calcarine sulcus $(r=0.457)$. This compares with a somewhat higher rate of $0.253 \pm 0.003 \mathrm{~mm}$ per millimeter distance from the PC for points on the right posterior ramus $(r=0.942)$, and a rate of $0.125 \pm 0.002 \mathrm{~mm}$ per millimeter distance from the $\mathrm{PC}$ for points on the right parieto-occipital sulcus $(r=0.846)$.

As for the paralimbic sulci, a relatively heterogeneous profile of variability was exhibited by the supracallosal sulcus in both hemispheres. The anterior terminus of the gray matter, which separates 
3D Stereotaxic Variability

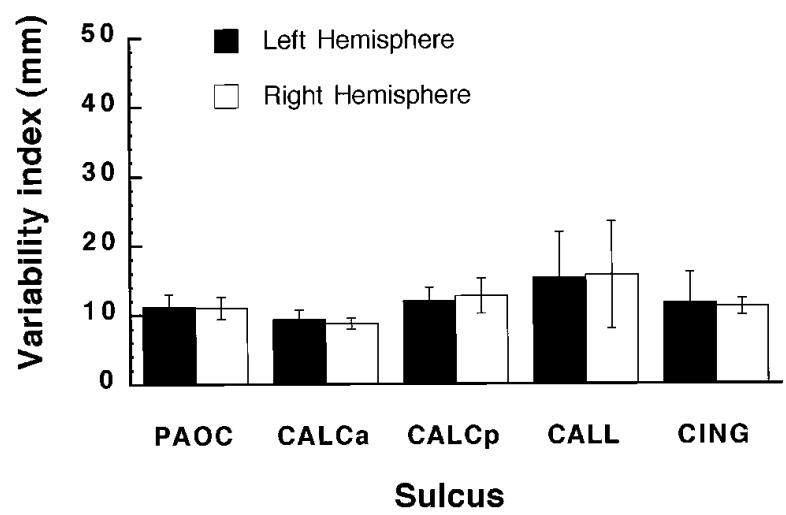

B Directional Components of Variability

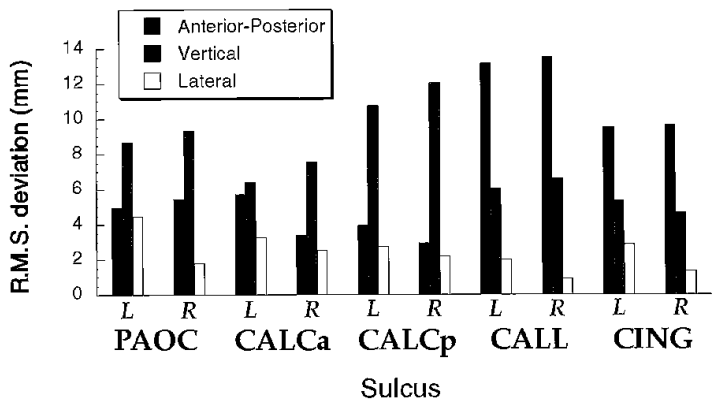

Figure 6. A, Sulcal variability expressed as a $3 \mathrm{D}$ distance in stereotaxic space. This summary measure of variability is obtained as follows. The map, which displaces the sulcal surface in a given specimen onto the average representation for that sulcus, assigns a $3 \mathrm{D}$ displacement vector to each node in the specimen surface. Comparison of the six specimen surface maps yields a variance value for the magnitude of the displacement vector assigned by each map to a given node. The square root of this measure gives the positional SD of each node as a distance in stereotaxic space. The mean and SD of these nodal values are shown here for each sulcus. This final numeric value gives a global indication of the stereotaxic variability of each sulcus when all the nodes on its surface are taken into account. Notice the relatively heterogeneous profile of variation exhibited by the callosal sulcus in both hemispheres. Rapidly changing profiles of the callosal genu were observed from one section to the next during delineation of this sulcus. This factor undoubtedly contributed to the high intersubject variance in the anterior segment of the structure. $B$, Resolution of sulcal variability into directional components. Displacement maps are used to encode the spatial relations of sulci in different individuals. These maps may then be analyzed into orthogonal components along each of the three axes of stereotaxic space. When the discrepancies among the sulci are considered separately along each orthogonal dimension of Talairach space, several directional biases become apparent. All six occipital sulci $(P A O C, C A L C a, C A L C p)$ vary most prominently in the vertical direction, whereas the four paralimbic sulci $(C A L L, C I N G)$ display the greatest variation in the anterior-posterior direction ( $L$ and $R$ denote structures in the left and right hemispheres, respectively). Lateral components of variability are consistently the lowest of all. Consequently, spatial variability in the internal anatomy of the sulci is not isotropic, exhibiting inherent directional biases characteristic of each individual sulcus.

the callosum from the cingulate gyrus, was regarded as defining the anterior limit of the supracallosal sulcus. The rapidly changing profiles of the superficial gray matter over the callosum, as observed during the segmentation of our data, undoubtedly contributed to the high intersubject variance at the anterior region of this structure.
The 3D rms variability for the cingulate sulcus in each hemisphere was not markedly correlated with radial distance from either of the commissures (all $r<0.37$ ). These sulci penetrate both the medial and frontal subvolumes of Talairach stereotaxic space, so that the Talairach transformation subjects different regions of their surfaces to different amounts of scaling (Fig. 5). This factor is likely to complicate any simple correlation between local variability of points on the cingulate surface and their distance to the stereotaxic control points.

\section{Contouring reliability}

The reliability of the contouring process itself was evaluated by repeatedly delineating the same structure and comparing the data obtained in multiple trials. All 10 structures in a single, randomly selected brain were manually outlined 6 times in random order. Outlines were converted to parametric mesh form, and the full range of geometric parameters was calculated for each surface. Results of these tests are presented in Tables 1 and 2. Curvature and fractal dimension measures were the most robust-worst-case errors represented 0.38 and $0.036 \%$ of the corresponding mean values for these measures [anterior calcarine sulcus (CALCa), Table 1]. Standard errors for repeated measures of extent and area data were, in the worst cases, only $0.32 \mathrm{~mm}$ and $0.030 \mathrm{~cm}^{2}$, respectively (parieto-occipital sulcus, Table 2). All measures were stable across the series of trials. The effects of contouring errors on each geometric variable were, in all cases but one, between 10 and 150 times smaller than the corresponding variation in the same quantity across the group of subjects. The worst case occurred when measuring the variability in curvature for the CALCa. Intersubject variability was, in this case, very small (SD $0.030 ; n=6$ ). Even so, this measure of variability between subjects was still a factor of 7 times greater than the effect of contouring error on this parameter (SD: $0.004 ; n=6$ ).

The regional impact of identification error and hand jitter during manual outlining was assessed in greater detail by creating additional 3D variability maps, showing local profiles of contouring error across each structure. The algorithms developed for calculating variability across subjects were used to map out local discrepancies, which occurred in contouring the same structure in

Table 1. Effect of contouring errors on curvature measures, surface complexity, and location in stereotaxic space

\begin{tabular}{llll} 
Structure & Curvature & Fractal dimension & $\begin{array}{c}\text { rMS nodal devia- } \\
\text { tion }(\mathrm{mm})\end{array}$ \\
\hline PAOC & $1.2072 \pm 0.0026$ & $\begin{array}{l}2.11117 \pm 0.00037 \\
(0.018 \%)\end{array}$ & $0.250 \pm 0.095$ \\
& $(0.22 \%)$ & $2.09433 \pm 0.00075$ & $0.190 \pm 0.082$ \\
CALCa & $1.0691 \pm 0.0040$ & $(0.036 \%)$ & \\
& $(0.38 \%)$ & $2.10183 \pm 0.00037$ & $0.235 \pm 0.064$ \\
CALCp & $1.2434 \pm 0.0024$ & $(0.018 \%)$ & \\
& $(0.19 \%)$ & $2.10933 \pm 0.00047$ & $0.341 \pm 0.136$ \\
CALL & $2.0548 \pm 0.0049$ & $(0.022 \%)$ & \\
& $(0.24 \%)$ & $2.12050 \pm 0.00050$ & $0.378 \pm 0.165$ \\
CING & $1.3469 \pm 0.0021$ & $(0.024 \%)$ &
\end{tabular}

This table summarizes the differences that occurred in outlining the same structure in the left hemisphere of a randomly selected brain specimen on multiple occasions $(n=6)$. Meshes were constructed from the outlines produced in different trials, and the rms nodal deviation measures summarize the 3D spatial discrepancies in the stereotaxic locations of their grid points, across the series of trials. All studies of morphometric variation across subjects incorporate identification errors as a source of variability. For each structure, mean measures and their SEs are given; SEs are also expressed as a percentage of the corresponding mean values. The low values suggest that contouring error represents a negligible fraction of the overall intersubject variability. 


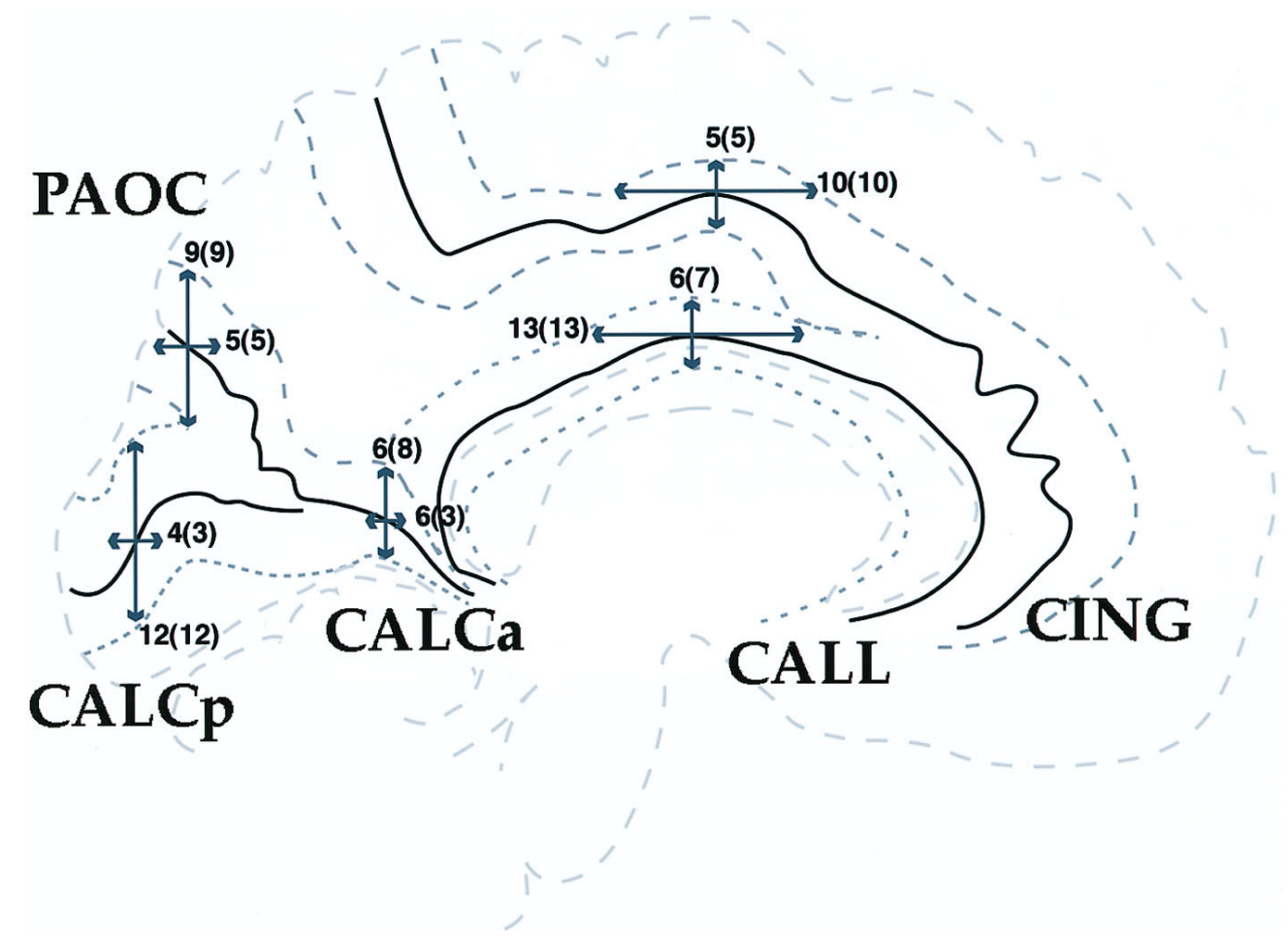

Figure 7. Inherent directional biases in sulcal variability. Components of sulcal variability in both the anterior-posterior and vertical directions are illustrated schematically (arrows) on a single sagittal section. Numerical values for these components, in millimeters, are also shown. For each pair of values given, the first value refers to structures in the left hemisphere; values in parentheses indicate structures in the right hemisphere. Confidence regions for structure identification are represented in the vicinity of each sulcus (internal dotted lines). Portions of sulci falling outside these designated regions are dislocated by $>1$ SD from the average sulcal surface in both of the chosen directions.

multiple trials $(n=6)$. Figure $8 C$ shows an example of such an error map for the three occipital sulci in the left hemisphere of the selected brain. In the left hemisphere, for example, contouring error across trials was smallest for the rather flat anterior branch of the calcarine sulcus (mean nodal deviation $0.190 \pm 0.082 \mathrm{~mm}$ ). This compared with a marginally higher error of $0.235 \pm 0.064$ $\mathrm{mm}$ for the posterior branch, $0.250 \pm 0.095 \mathrm{~mm}$ for the parietooccipital sulcus, and $0.341 \pm 0.136$ and $0.378 \pm 0.165 \mathrm{~mm}$ for the callosal and cingulate sulci, respectively. Although the magnitude of contouring error was confirmed to be small throughout, it was not uniform across the surface of each structure. In particular, greater error was observed in regions of high differential curvature (Fig. $8 C$ ). This battery of tests indicated that the variability in delineating sulcal trajectories represented a negligible fraction of the overall intersubject variability, which was consistently a factor of 30-80 times greater than the inherent errors in the contouring process.

\section{Surface curvature and fractal dimension}

Measures of surface curvature and fractal dimension are shown in Figure $10, A$ and $B$. As might be expected, surface curvature is higher for the arched paralimbic sulci than for those framing the gyri of the occipital lobe. Fractal dimension, however, is strikingly uncorrelated with surface curvature. This finding is consistent with the hypothesis that the architectonic surfaces bounding the sulci all exhibit local convolutions to approximately the same degree, regardless of their global conformation and overall intracerebral course.

\section{DISCUSSION}

\section{Heterogeneous profiles of 3D variation}

The sulcal mapping approach presented here provides a framework for structural analysis of the interior surface anatomy of the brain in three dimensions. A family of surface maps was constructed, encoding statistical properties of local anatomical variation within individual sulci.

Previous studies have examined the relationship between the locations of cortical landmarks as specified by an atlas and those found experimentally (Talairach et al., 1967; Missir et al., 1989; Steinmetz et al., 1989, 1990; Vannier et al., 1991). However, none has included an analysis of stereotaxic variation in $3 \mathrm{D}$ space. Nor have previous investigations analyzed variability into specific directional components, or ascertained whether there are any principal directions along which anatomic variation is greatest. Quantitative information has been limited by the identification difficulties and interslice resolution limits of MR imaging. Until very recently, investigations have also focused on defining the superficial course of sulci (Vannier et al., 1991) rather than the complex internal cytoarchitectural surfaces framed by the sulci in three dimensions (Rademacher, 1993).

The local measures of spatial variability, quantified here in three dimensions, agree in most respects with earlier investigations based on projecting sulcal outlines orthogonally onto a single plane (Talairach et al., 1967; Missir et al., 1989; Steinmetz et al., 1989, 1990; Vannier et al., 1991), but differ in other respects. In Steinmetz et al. (1990), maximal variation zones of 15-20 mm were recorded for sulci measured in series of $5 \mathrm{~mm}$ apart MR images. Because sulci were measured on the brain's exterior 

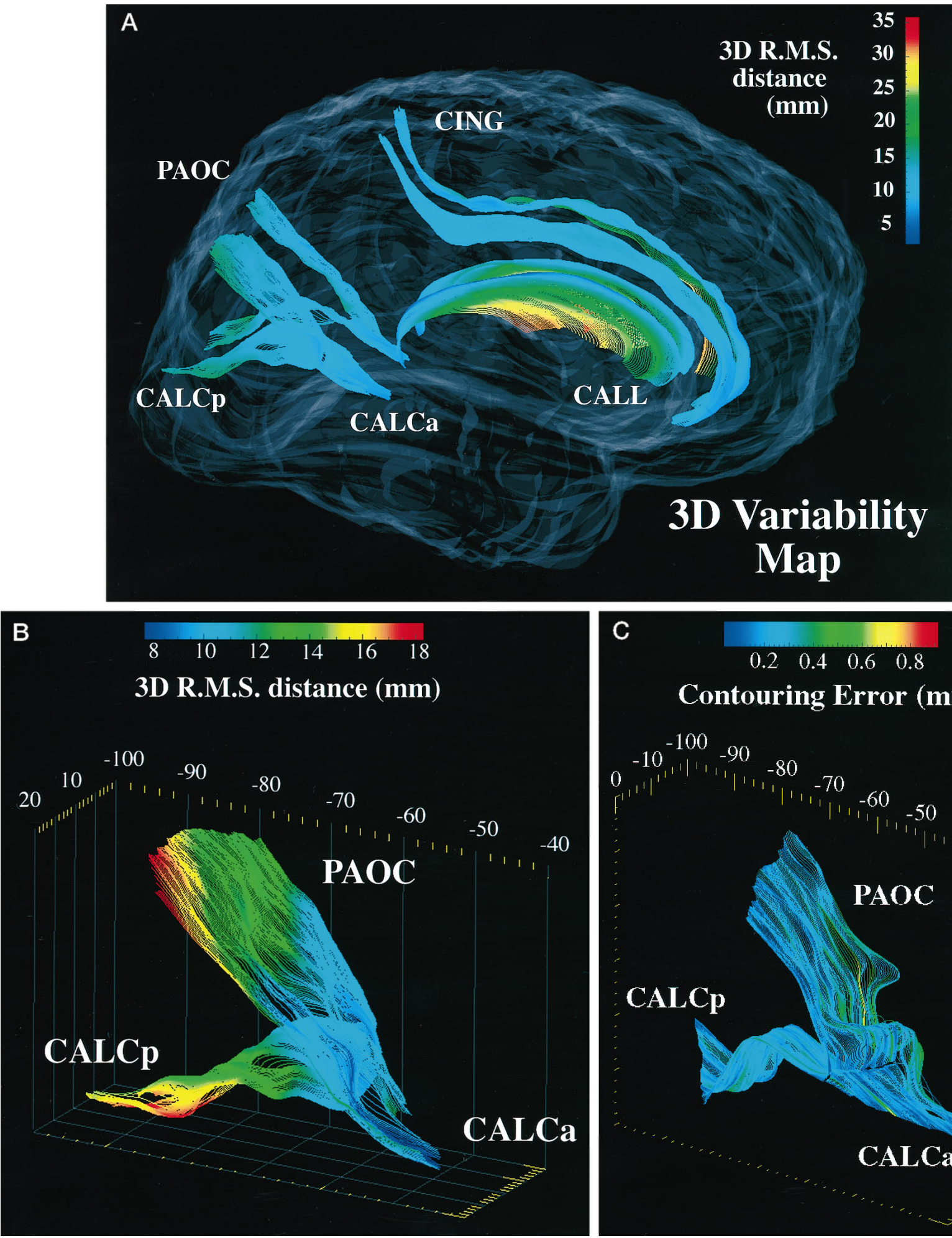

\section{Contouring Error (mm)}

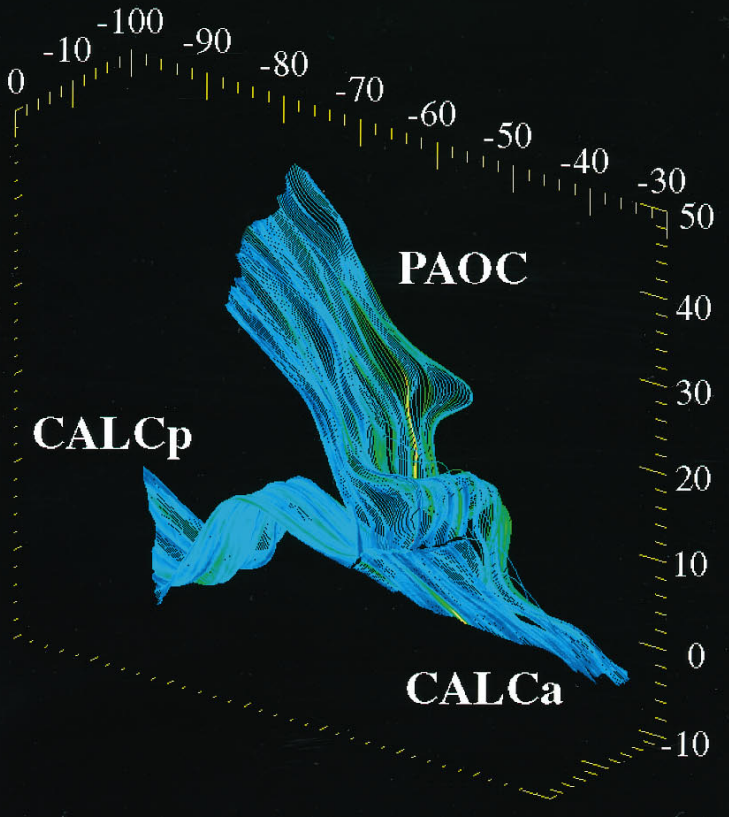

Figure 8. A, Average surface representations and 3D variability maps for major sulci in both hemispheres. 3D modeling and surface reconstruction techniques allow visualization of sulcal topography and greatly enhance the ability to appreciate complex spatial relationships. 3D representations are shown for all 10 average sulci from corresponding hemispheres of the six specimen brains. In this case, local variability is shown in color, on an average representation of each sulcus in Talairach stereotaxic space. The color encodes the rms magnitude of the displacement vectors required to map the surfaces from each of the six specimens onto the average, according to standard parametric criteria. $B, 3 \mathrm{D}$ variability maps for major sulci of the occipital lobe. This oblique right-hand side view illustrates the course of the parieto-occipital sulcus from its anteroventral junction with the medial surface of the calcarine sulcus, which it divides into anterior and posterior segments. The posterior calcarine sulcus is shown joining it inferiorly. Notice the pronounced increase in variability toward the exterior occipital surface. Such surface models can be rotated and magnified interactively by the viewer to enhance the appreciation of complex spatial relationships. $C$, Error maps showing reliability of structure delineation in multiple trials. The reliability of the contouring process was evaluated by repeatedly delineating the same structures in a randomly selected brain. Algorithms developed for calculating variability across subjects were used to map out local discrepancies, which occurred when contouring the same structure in multiple trials $(n=6)$. 3D surface models of the parieto-occipital, anterior, and posterior calcarine sulci are 


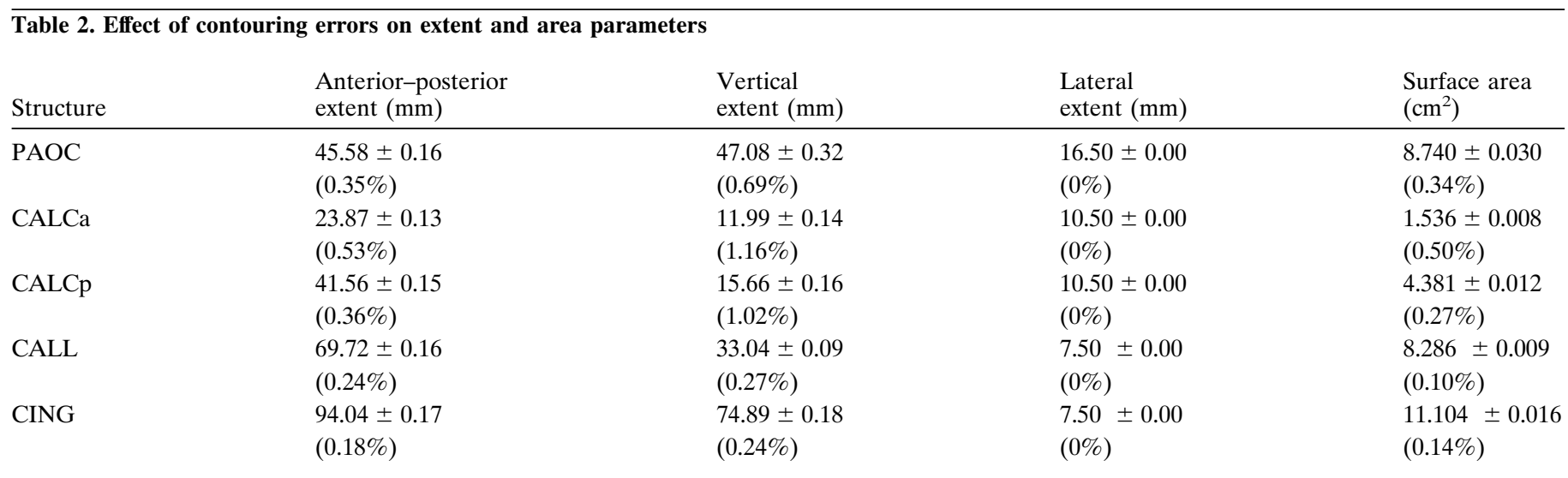

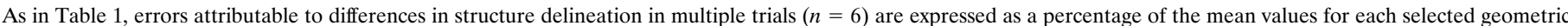

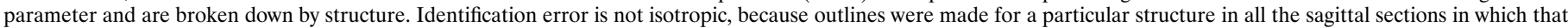

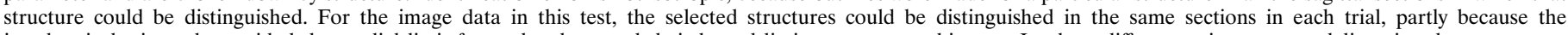

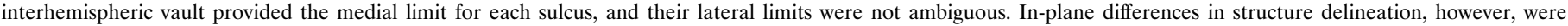
introduced across multiple trials, and these contributed to differences in the surface areas, as well as the vertical and rostral extents, of each individual structure.

surface, it was suggested that similar spatial variability must be assumed for the deeply located parts of the sulci. Our findings do not support this conclusion, especially with regard to the six occipital sulci. The parieto-occipital sulcus, as well as the anterior and posterior rami of the calcarine sulcus in each hemisphere, exhibit very marked increases in their variability with 3D distance from the posterior commissure. For the parieto-occipital, posterior calcarine, and cingulate sulci, the associated confidence limits on $3 \mathrm{D}$ variation increased from an SD of $8-10 \mathrm{~mm}$ internally to a peak of $17-19 \mathrm{~mm}$ at the exterior cerebral surface.

In spite of their numerical similarity, these measures of the $3 \mathrm{D}$ rms variation differ from the variability measures used in earlier studies (Talairach et al., 1967; Steinmetz et al., 1989, 1990) in two major respects. First, because previous studies projected sulcal outlines orthogonally onto a single plane, substantial components of spatial variability in the lateral dimension (Figs. 6B, 9C) were factored out. 3D rms measures, however, reflect variability in all spatial directions. Second, the existence of an underlying average surface representation for each sulcus allows parameters of dispersion, such as SDs and confidence limits, to be estimated directly from the distributions of each sulcal surface in stereotaxic space. 3D variability maps are also more robust indicators of spatial variation for anatomic structures than 2D maximal variation zones, because the latter measures are based solely on outliers (Talairach et al., 1967; Steinmetz et al., 1989, 1990).

Previous investigations have not specifically addressed the question of whether normal anatomic variations are spatially isotropic or whether they possess an inherent directionality. Decomposition of the variations in sulcal position into components along the three orthogonal axes of stereotaxic space reveals several underlying trends. First, the selected sulci are in general more likely to be found displaced in a vertical or anterior-posterior direction rather than laterally, relative to any fixed representation of neuroanatomy (Fig. 6B). Second, paralimbic sulci exhibit a greater degree of anterior-posterior variability than vertical variability, with the reverse trend being demonstrated by the occipital sulci (Fig. 9).

Heterogeneous profiles of variation in the internal surface geometry of the brain are the end product of an almost infinite variety of evolutionary, developmental, and experiential processes. Nevertheless, the intersubject variability documented here and in earlier studies may well be largely determined by two major factors.

\section{Developmental effects}

The differential arching of the limbic system during the embryonic process is accompanied by a dynamic regime of local deformations and differential growth throughout the material architecture of the brain (Toga et al., 1996). Differential growth in the caudate causes the gross anatomy of the brain to arch into a $\mathrm{C}$-shape between 2 and 5 months gestational age. This internal arching of the caudate and the fornix induces a similar geometric arching in the structures that surround them, including the cingulate and parahippocampal gyri of the limbic system. The entire cerebrum, anchored to this dynamically evolving foundation, also sustains changes in its surface geometry as a result, before the formation of fissures in the cerebral surface and the generation of the internal surface architecture of the sulci. Local differences in the rate of this angular deformation of cerebral tissue during development could create a pattern of anatomic variability across individuals, which results in large differences in relative extents, as well as differences in the local curvature and complexity of the mature sulcal pattern.

\section{The Talairach stereotaxic system}

Talairach stereotaxic space continues to be widely accepted by the neuroscience community as a precise quantitative framework for multimodality mapping (Fox et al., 1985; Evans et al., 1994b), as well as for coordinate-based morphometry and neurosurgical studies (Talairach et al., 1967; Burzaco, 1985; Missir et al., 1989;

derived from the left hemisphere of the randomly selected brain. The color encodes the rms magnitude of the displacement vectors required to map the surface obtained in each trial onto the average of the surfaces obtained in multiple trials. Notice that the color scale represents a range of variations 20 times smaller in magnitude than the intersubject variations shown in $B$. Note also the greater error in regions of higher differential curvature. Stability of individual geometric parameters across multiple trials, in conjunction with error maps of these and other structures, indicates that the variability in delineating sulcal trajectories represented a negligible fraction of the overall variability between subjects. 
A

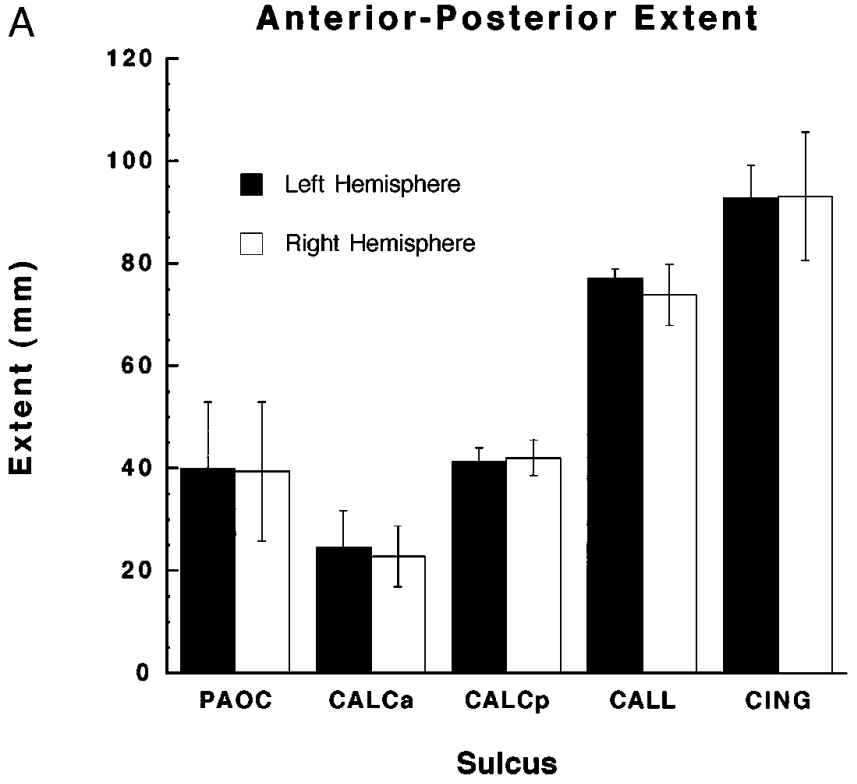

C

כ)
B

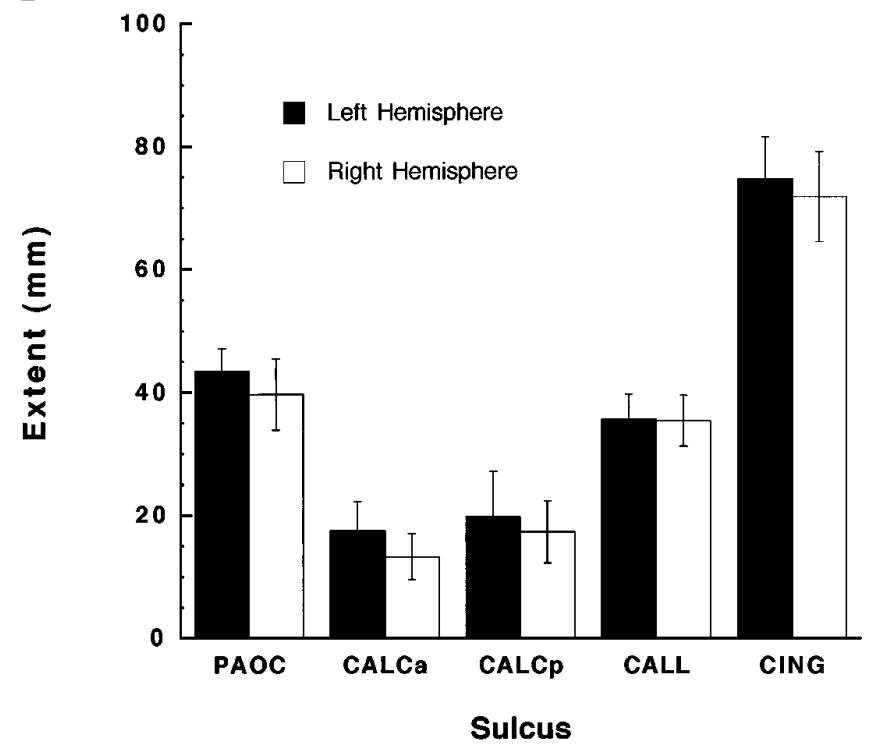

D

Surface Area

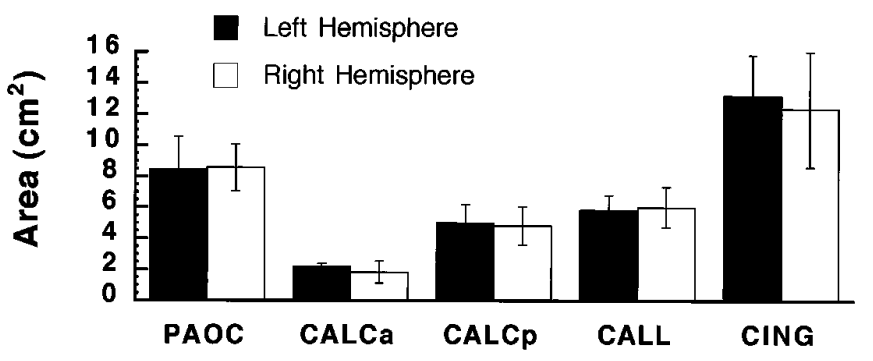

Sulcus

Sulcus

Figure 9. $A-D$, Stereotaxic extents of sulcal surfaces and their surface areas. These graphs illustrate the overall trends in spatial extent and area for the sulcal surfaces under examination. $A-C$, The total extent of each sulcus along each orthogonal dimension of Talairach space was measured in both left and right hemispheres. Error bars indicate SD measures. Note the marked symmetry of results for both hemispheres, as expected for the structures examined. Surface area measures are illustrated in $D$.

Steinmetz et al., 1989, 1990; Vannier et al., 1991; Mazziotta et al., 1995). However, the pronounced residual variations in the stereotaxic position of cortical landmarks, as quantified by this study and reported in earlier investigations, underscore the significant limitations of the Talairach system in localizing cortical structures. Stereotaxic systems differ significantly in their capacity to compensate for intersubject variations in the anatomy of the brain (Burzaco, 1985). As documented in this study, significant morphometric variability remains after transformation of brain data into Talairach stereotaxic space, and these variations exhibit significant geometric and directional biases. The two 3D control points for the rectangular affine normalization are defined as the
AC-PC points, near the center of the brain, and these points are assigned canonical locations in stereotaxic space. There is therefore no intersubject anatomical variation at these points, and increasing variability further away from them. Although the variability across sulcal surfaces increases toward the external cortex, the outer cortex itself is constrained to occupy a fixed rectangular bounding box of a specific size. The calcarine sulcus, for example, is bounded anteriorly by the PC point and posteriorly by the back of the brain. Its variability is therefore constrained in the anteriorposterior direction, whereas it is less restricted in the vertical direction, and accordingly exhibits a greater variance. Similar arguments apply to the other sulci. The consistently low lateral 


\section{A Normalized Curvature Index}

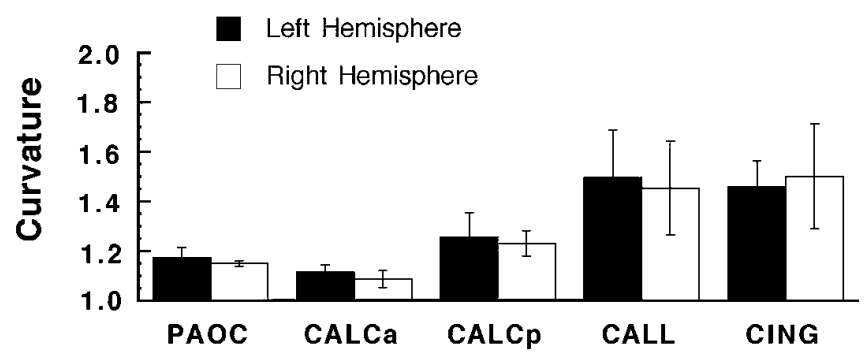

Sulcus

B Fractal Dimension

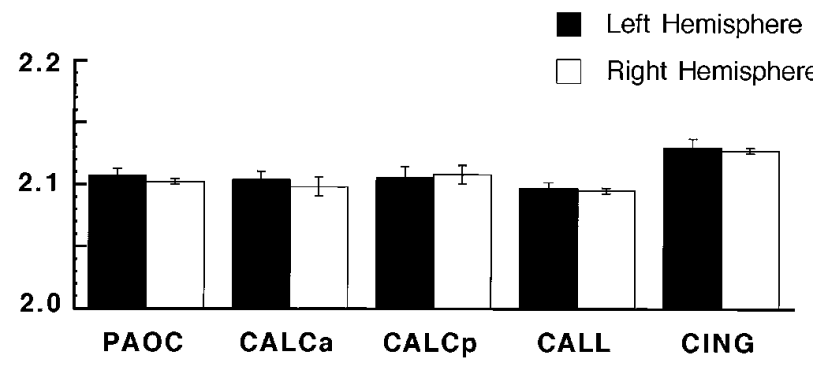

Sulcus

Figure 10. $A, B$, Indices of normalized curvature and fractal dimension for each sulcus. Trends in surface curvature and geometric complexity are shown for each sulcus. Fractal dimension is an extremely compact measure of surface complexity, condensing all the details of surface shape into a single numeric value, which summarizes the irregularity of the sulcal course inside the brain. Briefly, the measure reflects the rate at which the surface area of the sulcus increases as the scale of measurement is reduced. Despite differences in surface curvature, the paths into the brain of all of the primary sulci examined are strikingly similar in complexity.

components of variability for the major sulci examined here may well reflect their proximity to the interhemispheric fissure, the position of which is fixed in the Talairach system. Any system for coordinate-based morphometry is likely to be effective in reconciling population variances of structures close to its control points (Steinmetz et al., 1989). Statistical sulcal mapping therefore may be used as a metric to evaluate different stereotaxic systems and to compare their effectiveness in reconciling intersubject variations.

\section{Cryosectioning}

As an overall strategy for quantifying differences in the neuroanatomy of human subjects, an approach based on rapid cryosectioning, in conjunction with high-resolution digital imaging of the specimen, presents certain specific advantages and disadvantages. Several of these factors deserve to be emphasized. First, the composition of the sample group in this study was inevitably constrained by the inherent difficulties in acquiring high-quality, normal anatomic specimens from a Willed Body Program. Nevertheless, strict exclusion criteria were applied to guarantee, as far as possible, the selection of normal brains. No significant quanti- tative differences have been found for any of the selected spatial and geometric parameters between formalin-treated and untreated specimens. The retention of the intact calvarium, rapid freezing, and the application of advanced cryoprotectants serve to directly counteract any distortion of the gross anatomy of the brain (Mega et al., 1995; Toga et al., 1995). Furthermore, the Talairach system specifies a rescaling of all brain specimens to the same extent along all three coordinate axes.

Second, any comparative analysis of anatomy in any species must be based on a sample of subjects whose ages are carefully controlled. Although measures were taken to restrict this analysis to a sample of relatively aged subjects, further investigations are required to determine whether the spectrum of variation documented here is reflected in other age groups and whether the selected surface measures are in any way a function of atrophy or age.

Finally, whole-brain imaging at this resolution requires the methodical application of a set of precise $3 \mathrm{D}$ anatomic criteria for delineating individual sulci. The $3 \mathrm{D}$ course of each primary sulcus into the brain was defined to be the medial surface equidistant from each opposing gyral bank (cf. Széleky et al., 1992). This medial axis definition provides the basis for a descriptive hierarchy in that it ascribes a fundamental laminar path into the brain for each primary sulcus. Analysis of the complex internal topology of the sulci is simplified considerably if the locations of secondary branches are directly referable to a single surface representation for the primary sulcus. Secondary parameters of variation, such as the local width and depth of the sulcus, can also be described more effectively if they are viewed as variables that depend on position relative to the medial surface. Although the medial axis definition was specifically designed to be invariant to intersubject differences in the width of the internal sulci, which might be pronounced in an aged population, further analysis of sulcal width and other secondary parameters of variation are necessary.

In spite of the logistic difficulties, cryosectioning procedures present a number of highly advantageous features not available in the clinical imaging modalities. Cryosectioning can be combined with a wide variety of molecular and neurochemical techniques on harvested tissue sections to enable parallel or subsequent characterization of regional anatomy at a very fine structural level. Comprehensive studies have revealed striking intersubject and interhemispheric variations in the distributions of primary neocortical fields, and have clarified their relation to sulcal anatomy (Rademacher et al., 1993; Rajkowska and Goldman-Rakic, 1995). Accordingly, high-resolution images of cryosectioned human anatomy not only provide the necessary spatial and densitometric resolution for accurate morphometry, but also enable parallel or subsequent analysis of cellular fields and their molecular composition.

\section{Future directions}

In the future, sulcal mapping is likely to be fundamental to multisubject atlasing and many other brain mapping projects. 3D mapping of anatomic variability, in combination with methods for rapidly calculating an inventory of relevant geometric parameters, offers a framework for analyzing cortical variation in human subjects and provides a basis for discriminant analysis in pathological populations. Striking decreases in the fractal dimension of the cerebral cortex have been associated with neurodegenerative diseases such as epilepsy (Cook et al., 1994), reflecting a dramatic reduction in the complexity of the cortical surface. Fractal dimension has been widely used as an indicator of surface complexity in 
biological systems, describing the degree of structural detail in bronchial and vascular trees, as well as the cerebral cortex (Cook et al., 1994; Griffen, 1994), and many other objects with convoluted geometry (Cressie, 1991; Stoyan and Stoyan, 1994). The relative invariance of this measure in our sample of normal brains suggests that a stable baseline exists, offering scope for further comparisons with pathological specimens in which this measure may be depressed. Global surface descriptors such as fractal dimension and curvature provide a means for highlighting subtle and distributed variations in anatomic structure, which may not be appreciated by visual inspection alone.

In addition, many sulci penetrate sufficiently deeply into the brain to introduce a topological decomposition of its volume architecture. Deep sulci, therefore, are natural control surfaces to choose (especially in parametric mesh form) as a basis for driving warping algorithms, which deform brain images locally and thereby integrate intersubject brain data (Toga, 1994; Joshi et al., 1995; Davatzikos et al., 1996; Thompson and Toga, 1996).

Atlasing considerations suggest that a confidence limit, rather than an absolute representation of neuroanatomy, may be more appropriate for representing a given subpopulation. A digital anatomic atlas of the human brain, incorporating precise statistical information on positional and geometric variability of important functional and anatomic interfaces, may present a convenient solution (Mazziotta et al., 1995). Elegant approaches exist for generating average representations of brain anatomy by densitometric averaging of multiple MR image volumes (Evans et al., 1992a; Andreasen et al., 1994). Nevertheless, the average brains that result have regions (especially at the cortical surface) where individual structures are blurred out because of spatial variability in the population, making them insufficient as a quantitative tool (Evans et al., 1994b).

Parametric mesh-based approaches, when generalized to encompass a sufficiently large set of architectonic surfaces in the brain, may offer distinct advantages over volume averaging for statistical atlasing applications (Thompson et al., 1996). These methods can be used to characterize simultaneously the spatial and geometric variation across different individuals of multiple, complex, branching, and arbitrarily connected anatomic surfaces in the brain. More specifically, however, the averaging procedure itself does not lead to the same type of degradation of structural geometry (and loss of fine anatomic features) as is often apparent in volume-averaging approaches. Finally, the retention of an explicit surface topology after averaging is particularly advantageous for subsequent visualization in that it permits the display of secondary parameters such as local variance in the form of a color-coded relief map on the resulting set of surfaces (Sclaroff, 1991; Thompson et al., 1996).

The ultimate goal of brain mapping is to provide a framework for integrating functional and anatomical data across many subjects and modalities. This task requires precise quantitative knowledge of the variations in geometry and location of intracerebral structures and critical functional interfaces. The surface mapping results presented here provide a basis for the generation of anatomical templates and for future analyses of structural variability in the human brain.

\section{APPENDIX}

Mathematical and cytoarchitectural considerations suggest that parametric mapping of architectonic surfaces offers a powerful method for representing complex associations between subregions of surfaces with subtle differences in geometry. A parametric approach to representing and mapping neuroanatomic surfaces has been fundamental to several recent advances in the notion of mapping the cerebral cortex. Cortical flattening algorithms, for example, are based on an explicit parameterization of the cortical surface (Van Essen and Maunsell, 1980; Carman et al., 1995). Parametric mesh approaches, which define a mapping of a 2D regular grid onto a complex 3D surface (Pedersen, 1994), were developed in a study by Bookstein et al. (1985) as a quantitative method for comparing biological shape across subjects. Parametric strategies have been validated as a paradigm for analysis of the cortical surface (MacDonald et al., 1993; Griffen, 1994; Bookstein, 1995). They were recently used to generate a high-dimensional probabilistic representation of brain structure, capable of detecting and quantifying subtle and distributed abnormalities in the anatomy of new subjects (Thompson et al., 1996). Parametric surface models of the cortex have also formed the basis of boundary-based warping algorithms, which integrate neuroanatomic data from subjects with different brain geometry (Joshi et al., 1995; Davatzikos et al., 1996; Thompson and Toga, 1996). In particular, the explicit geometry provided by this approach allows convenient derivation of morphometric statistics, as well as quantitative indices of surface curvature, extent, area, fractal dimension, and geometric complexity.

\section{Parametric mesh construction}

Strategies for creating a regular parametric mesh from a stack of sulcal outlines contoured in a series of sagittal sections are analogous to stretching a regular rectangular grid, of size $I \times J$ (for any integers $I$ and $J$ ), over all the scattered 3D point data digitized when outlining the sulcus (Fig. 3). The mesh of grid points that results is parametric in the sense that its nodes can be indexed using the coordinates of the superimposed grid, $u$ and $v$, where $u$ and $v$ are non-negative integers. $u$ and $v$ are then said to be parametric coordinates for points on the surface. 3D surface points are given as position vectors in Talairach space by the function $\mathbf{r}_{u}^{v}$ $=(x(u, v), y(u, v), z(u, v))$, (q.v., Fig. 3). Full technical details of the mesh construction algorithm are presented in Thompson et al. (1996). Briefly, manual outlining of each sulcal surface $S$ produces a set of parallel cross-sections $C_{0}, C_{1}, C_{2}, \ldots, C_{K}$ of $S$, at $z_{0}, z_{1}$, $z_{2}, \ldots, z_{K}$, where $z$ is the lateral axis of Talairach space. Each contour is itself a set of $3 \mathrm{D}$ digitized points $C_{k}=\left\{P_{i}\left(x_{i}^{k}, y_{i}^{k}, z_{i}^{k}\right) \mid 0\right.$ $\left.\leq i \leq N_{k}\right\}$, where the number of points in each contour, $N_{k}$, varies for different contours, $C_{k}$, in the stack. Let $\|\mathbf{x}-\mathbf{y}\|$ denote the distance between $3 \mathrm{D}$ points $\mathbf{x}$ and $\mathbf{y}$. To create a mesh of size $I \times$ $J$, we first define, for each $C_{k}$, a cumulative arc length $l\left(\mathbf{p}_{i}^{k}\right)=\Sigma_{j=1}$ to $i\left\|\mathbf{p}_{j}^{k}-\mathbf{p}_{j-1}^{k}\right\|$ to point $\mathbf{p}_{i}^{k}=P_{i}\left(x_{i}^{k}, y_{i}^{k}, z_{i}^{k}\right)$. For each integer $u=0$ to $I$, we also let $i(u)=\min \left\{i \mid l\left(\mathbf{p}_{i}^{k}\right)>u \cdot l\left(\mathbf{p}_{N k}^{k}\right) / I\right\}$. A family of $k$ parametric curves is then given by $\mathbf{q}_{u}^{k}=\mathbf{p}_{i(u)-1}^{k}+\lambda\left(\mathbf{p}_{i(u)}^{k}-\right.$ $\left.\mathbf{p}_{i(u)-1}^{k}\right)$, where $\lambda=\left\{\left(u \cdot l\left(\mathbf{p}_{N k}^{k}\right) / I\right)-l\left(\mathbf{p}_{i(u)-1}^{k}\right)\right\} /\left\{l\left(\mathbf{p}_{i(u)}^{k}\right)-\right.$ $\left.l\left(\mathbf{p}_{i(u)-1}^{k}\right)\right\}$. We then let $l_{u}\left(\mathbf{q}_{u}^{k}\right)$ be the cumulative arc length $\Sigma_{i=1 \text { to } k}\left\|\mathbf{q}_{u}^{i}-\mathbf{q}_{u}^{i-1}\right\|$. For each integer $v=0$ to $J$, we let $i(v)=$ $\min \left\{i \mid l_{u}\left(\mathbf{q}_{u}^{i}\right)>v \cdot l_{u}\left(\mathbf{q}_{K}^{i}\right) / J\right\}$ and $\mu=\left\{\left(v \cdot l_{u}\left(\mathbf{q}_{K}^{i}\right) / J\right)-l_{u}\left(\mathbf{q}_{u}^{i(v)-1}\right)\right\} /$ $\left\{l_{u}\left(\mathbf{q}_{u}^{i(v)}\right)-l_{u}\left(\mathbf{q}_{u}^{i(v)-1}\right)\right\}$. Then the 3D lattice of points $\mathbf{r}(u, v)=$ $\mathbf{q}_{u}^{i(v)-1}+\mu\left(\mathbf{q}_{u}^{i(v)}-\mathbf{q}_{u}^{i(v)-1}\right), 0 \leq u \leq I, 0 \leq v \leq J$, designates the grid points of a regular parametric mesh of size $I \times J$ spanning the sulcal surface $S$.

\section{Use of meshes to define point correspondences}

Under certain strict conditions, the imposition of regular grids onto 3D biological surfaces permits cross-subject comparisons by association of different kinds of points (landmark and nonlandmark) among geometric forms (Bookstein et al., 1985). They 
define a surface-based coordinate system (Fig. 3) that specifies a computed correspondence along the outline arcs and within the interior of the structures (Bookstein, 1985). For the comparisons to be valid, landmark points and curves known to the anatomist must appear in corresponding locations in each parametric grid. For this reason, the calcarine sulcus (Fig. $8 A-C$ ) was not modeled as a single mesh, but was partitioned into two meshes (CALCa and CALCp). The complex 3D curve forming their junction with the parieto-occipital sulcus therefore was accurately mapped under the displacement maps, which relate one anatomy to another. Our landmark constraints on sulcal parameterizations included the partitioning of parametric elements along landmark curves known to the anatomist. Nevertheless, we did not regard the genu or splenium of the corpus callosum as appropriate landmarks for constraining the parameterization of the callosal sulcus, because, as noted in Bookstein (1985, p.6), "it is not sufficient to choose, as landmarks, points having extreme values relative to a particular coordinate system." However, experiments were conducted to validate the accuracy of the mesh procedure in correctly associating the front of the callosal genu and the back of the splenium across subjects. We defined the genu and splenium in the average and specimen callosal sulci to contain the two sulcal points with extremal anterior-posterior coordinates in each sagittal section. These points were connected to produce a set of four reference curves in each brain, representing the genu and splenium in each hemisphere. Parametric estimates of the same structures were also independently defined, as the curves on the specimen meshes with the same parametric coordinates as the genu and splenium of the average surface. These two definitions were compared in six brains, and rms distances between the two defined curves were calculated. The parametric estimates of the left genu were, on average, $0.45 \pm 0.34 \mathrm{~mm}$ from the true left genu when the discrepancy was measured along the anterior-posterior axis. This compared with a similar $0.44 \pm 0.28 \mathrm{~mm}$ discrepancy for the right genu, and $0.14 \pm 0.08$ and $0.19 \pm 0.06 \mathrm{~mm}$ for the left and right splenium, respectively. These results, together with the partitioning strategy outlined above, suggest that any errors attributable to homological misalignment of the meshes on different specimens represented a negligible fraction of the overall anatomical variability between subjects.

\section{Measures of curvature and fractal dimension}

For each type of sulcus represented as a parametric mesh $\left\{\mathrm{r}_{u}^{v} \mid 0\right.$ $\leq u \leq I, 0 \leq v \leq J\}$ of fixed size $I \times J$, a simple measure of surface curvature is given by $\operatorname{Curv}\left(\left\{\mathbf{r}_{u}^{v}\right\}\right)=\left\{\Sigma_{v=0 \text { to } J}\left\|\mathbf{r}_{I}^{v}-\mathbf{r}_{0}^{v}\right\|\right\} /$ $\left\{\Sigma_{v=0 \text { to } J} \Sigma_{u=1 \text { to } I}\left\|\mathbf{r}_{u}^{v}-\mathbf{r}_{u_{-1}}^{\mathrm{v}}\right\|\right\}$. This formula can be explained as follows. For any given slice in which a sulcal contour appears, the cumulative arc length, measured along the contour, exceeds the direct length of a hypothetical straight line joining the contour's end points. Similarly, for each of the grid lines in the mesh, this length excess can be expressed as a ratio, which reflects the degree of inherent curvature in the surface along that grid line. The normalized curvature index $\operatorname{Curv}\left(\left\{\mathbf{r}_{u}^{v}\right\}\right)$ is a more general ratio, which takes all grid lines into account. Its value is given by adding up the arc lengths along every grid line and dividing the total by the sum of the direct lengths of straight lines joining each of the grid lines' end points. Finally, an ordered hierarchy of parametric meshes $\left\{\mathbf{M}_{I J}\right\}$ was generated for each sulcus $S$, with variable resolution $I \times J(I=2$ to 100$)$. If $\mathbf{A}\left\{\mathbf{M}_{I J}\right\}$ represents the surface area of the mesh $\mathbf{M}_{I J}, S$ has fractal dimension $\operatorname{Dim}_{\mathrm{F}}(S)=2-\{\partial$ $\left.\ln \mathbf{A}\left\{\mathbf{M}_{I J}\right\} / \partial \ln (1 / I)\right\}$. The gradient of the associated multifractal plot can be obtained by least-squares regression of the function $\ln \mathbf{A}\left\{\mathbf{M}_{I J}\right\}$ against $\ln (1 / I)$, over the range $2 \leq I \leq 100$.

\section{Surface averaging and local variability measures}

Each type of sulcus, in either hemisphere, is contoured in six specimens. The mesh construction procedure therefore yields six regular parametric meshes $\mathbf{s}_{i}(u, v),(i=1$ to 6$)$ for each sulcus. The average sulcal surface is then given by another mesh of the form:

$$
\mu(u, v)=(1 / 6) \sum_{i=1 \text { to } 6} \mathbf{s}_{i}(u, v), \quad(0 \leq u \leq I, 0 \leq v \leq J) .
$$

The variability in stereotaxic position for points internal to a sulcal surface is then given by the scalar variance function:

$$
\begin{gathered}
\mathbf{s}^{2}(u, v)=(1 / 6) \sum_{i=1 \text { to } 6}\left\|\mathbf{s}_{i}(u, v)-\mu(u, v)\right\|^{2}, \\
(0 \leq u \leq I, 0 \leq v \leq J) .
\end{gathered}
$$

The square root of this function yields an estimate of the SD in stereotaxic position for each internal point on the surface. The values of this function are in Talairach millimeters, and their range can be linearly mapped via a look-up table onto a color range. Profiles of local variability can therefore be visualized, as they vary across each sulcal surface.

\section{REFERENCES}

Andreasen NC, Arndt S, Swayze V, Cizadlo T, Flaum M, O'Leary D, Ehrhardt JC, Yuh WTC (1994) Thalamic abnormalities in schizophrenia visualized through magnetic resonance image averaging. Science 266:294-298.

Bailey P, von Bonin G (1951) The isocortex of man. Urbana: University of Illinois.

Bookstein FL (1995) Proposal for a biometrics of the cortical surface: a statistical method for relative surface distance metrics. In: Proceedings of the SPIE Conference on Vision Geometry, Vol 2573, pp 312-323.

Bookstein F, Chernoff B, Elder R, Humphries J, Smith G, Strauss R (1985) Morphometrics in evolutionary biology, Special publication 15. Academy of Natural Sciences of Philadelphia.

Burzaco J (1985) Stereotaxic pallidotomy in extrapyramidal disorders. Appl Neurophysiol 48:283-287.

Carman GJ, Drury HA, Van Essen DC (1995) Computational methods for reconstructing and unfolding the cerebral cortex. Cereb Cortex 5:506-517.

Cook MJ, Free SL, Fish DR, Shorvon SD, Straughan K, Stevens JM (1994) Analysis of cortical patterns. In: Magnetic resonance scanning and epilepsy (Shorvon SD, ed), pp 263-274. New York: Plenum.

Cressie NAC (1991) Statistics for spatial data. New York: Wiley.

Davatzikos C, Prince JL, Bryan RN (1996) Image registration based on boundary mapping. IEEE Transactions on Medical Imaging 15:212-215.

Evans AC, Collins DL, Milner B (1992a) An MRI-based stereotactic brain atlas from 300 young normal subjects. In: Proceedings of the $22 \mathrm{nd}$ Symposium of the Society for Neuroscience, Vol 408, Anaheim, CA.

Evans AC, Marrett S, Neelin P, Collins L, Worsley K, Dai W, Milot S, Meyer E, Bub D (1992b) Anatomical mapping of functional activation in stereotaxic coordinate space. NeuroImage 1:43-54.

Evans AC, Collins DL, Neelin P, MacDonald D, Kamber M, Marrett TS (1994a) Three-dimensional correlative imaging: applications in human brain mapping. In: Functional neuroimaging: technical foundations (Thatcher RW, Hallett M, Zeffiro T, Roy John E, Huerta M, eds), pp 145-161. New York: Academic.

Evans AC, Kamber M, Collins DL, MacDonald D (1994b) An MRI-based probabilistic atlas of neuroanatomy. In: Magnetic resonance scanning and epilepsy (Shorvon SD, ed), pp 263-274. New York: Plenum.

Evans AC, Collins DL, Holmes CJ (1996) Computational approaches to quantifying human neuroanatomic variability. In: Brain mapping: the methods (Toga AW, Mazziotta JC, eds). New York: Academic, in press.

Fox PT, Perlmutter JS, Raichle ME (1985) A stereotactic method of anatomic localization for positron emission tomography. $\mathrm{J}$ Comput Assist Tomogr 9:513-530. 
Griffen LD (1994) The intrinsic geometry of the cerebral cortex. J Theor Biol 166:261-273

Joshi SC, Miller MI, Christensen GE, Banerjee A, Coogan T, Grenander U (1995) Hierarchical brain mapping via a generalized Dirichlet solution for mapping brain manifolds. In: Proceedings of the SPIE Conference on Geometric Methods in Applied Imaging, Vol 2573, pp 278-289.

MacDonald D, Avis D, Evans AC (1993) Automatic parameterization of human cortical surfaces. Annual Symp Info Proc Med Imag (IPMI).

Martin J, Pentland A, Kikinis R (1994) Shape analysis of brain structures using physical and experimental models. Proceedings of the IEEE Comput Soc Conference on Computer Vision and Pattern Recognition, June 21-23, Los Alamitos, CA.

Mazziotta JC, Toga AW, Evans AC, Fox P, Lancaster J (1995) A probabilistic atlas of the human brain: theory and rationale for its development. NeuroImage 2: 89-101.

Mega MS, Karaca TJ, Pouratain N, Chen S, Adamson CF, Schluender S, Toga AW (1995) Premortem-postmortem neuroimaging: a study of brain morphometric changes in man with MRI and cryomacrotome imaging. In: Proceedings of the 25th Symposium of the Society for Neuroscience, November 11-16, San Diego.

Missir O, Dutheil-Desclercs C, Meder JF, Musolino A, Fredy D (1989) Central sulcus patterns at MRI. J Neuroradiol 16:133-144.

Ono M, Kubik S, Abernathey CD (1990) Atlas of the cerebral sulci. Stuttgart: Thieme.

Pedersen HK (1994) Displacement mapping using flow fields. In: Proceedings of the SIGGRAPH Computer Graphics Conference, pp 279-286.

Quinn B, Ambach K, Toga AW (1993) Towards a digital reconstruction human brain atlas. In: Proceedings of the 23rd Symposium of the Society for Neuroscience, Vol 18, p 968.

Rademacher J, Caviness Jr VS, Steinmetz H, Galaburda AM (1993) Topographical variation of the human primary cortices: implications for neuroimaging, brain mapping and neurobiology. Cereb Cortex 3:313-329.

Rajkowska G, Goldman-Rakic P (1995) Cytoarchitectonic definition of pre-frontal areas in the normal human cortex. II. Variability in locations of areas 9 and 46 and relationship to the Talairach coordinate system. Cereb Cortex 5:323-337.

Roland PE, Zilles K (1994) Brain atlases-a new research tool. Trends Neurosci 17:458-467.

Ruprecht D, Nagel R, Muller H (1995) Spatial free-form deformation with scattered data interpolation methods. Computers and Graphics: 19:63-71.

Sanides F (1962) Die Architektonik des menschlichen Stirnhirns. In: Monographien aus dem Gesamtegebiete der Neurologie und Psychiatrie. (Mueller M, Spatz H, Vogel P, eds). Berlin: Springer.

Sclaroff S (1991) Deformable solids and displacement maps: a multiscale technique for model recovery and recognition. Master's thesis, MIT Media Laboratory.

Sclaroff S, Pentland A (1994) On modal modeling for medical images: underconstrained shape description and data compression. In: Proceedings of the IEEE Workshop on Biomedical Image Analysis, June 24-25, Los Alamitos, CA.
Steinmetz H, Furst G, Freund H-J (1989) Cerebral cortical localization: application and validation of the proportional grid system in MR imaging. J Comput Assist Tomogr 13:10-19.

Steinmetz H, Furst G, Freund H-J (1990) Variation of perisylvian and calcarine anatomic landmarks within stereotaxic proportional coordinates. Am J Neuroradiol 11:1123-1130.

Stensaas SS, Eddington DK, Dobelle WH (1974) The topography and variability of the primary visual cortex in man. J Neurosurg 40:747-755.

Stoyan D, Stoyan H (1994) Fractals, random shapes, and point fields: methods of geometrical statistics (Stoyan D, Stoyan H, eds). New York: Wiley.

Széleky G, Brechbühler C, Kübler O, Ogniewicz R, Budinger T (1992) Mapping the human cerebral cortex using 3D medial manifolds. In: Proceedings of the SPIE Conference on Visualization in Biomedical Computing, Vol 1808, pp 130-143.

Talairach J, Tournoux P (1988) Co-planar stereotaxic atlas of the human brain. New York: Thieme.

Talairach J, Szikla G, Tournoux P, Prosalentis A, Bordas-Ferrier M, Covello L, Iacob M, Mempel E (1967) Atlas d'anatomie stereotaxique du telencephale. Paris: Masson.

Thompson PM, Toga AW (1996) Mapping the internal cortex: a probabilistic brain atlas based on high-dimensional random fluid transformations. Human Brain Mapping, in press.

Thompson PM, Schwartz C, Toga AW (1996) High-resolution random mesh algorithms for creating a probabilistic 3D surface atlas of the human brain. NeuroImage 3:19-34.

Toga AW, Ambach KL, Quinn B, Hutchin M, Burton JS (1994a) Post mortem anatomy from cryosectioned human brain. J Neurosci Methods $54: 239-252$.

Toga AW, Ambach KL, Schluender S (1994b) High-resolution anatomy from in situ human brain. NeuroImage 1:334-344.

Toga AW (1994) Visualization and warping of multi-modality brain imagery. In: Functional neuroimaging: technical foundations (Thatcher RW, Hallett M, Zeffiro T, Roy John E, Huerta M, eds), pp 171-180. New York: Academic.

Toga AW, Ambach KL, Quinn B, Shankar K, Schluender S (1995) Post mortem anatomy. In: Brain mapping: the methods Chap 7, (Toga AW, Mazziotta JC, eds), pp 169-190. New York: Academic.

Toga AW, Thompson PM, Payne BA (1996) Modeling morphometric changes of the brain during development. In: Developmental neuroimaging: mapping the development of brain and behavior (Thatcher RW, Lyon GR, Rumsey J, Krasnegor N, eds). New York: Academic, in press.

Van Essen DC, Maunsell JHR (1980) Two-dimensional maps of the cerebral cortex. J Comp Neurol 191:255-281.

Vannier MW, Brunsden BS, Hildebolt CF, Falk D, Cheverud JM, Figiel GS, Perman WH, Kohn LA, Robb RA, Yoffie RL, Bresina SJ (1991) Brain surface cortical sulcal lengths: quantification with threedimensional MR imaging. Radiology 180:479-484.

Watson JDG, Myers R, Frackowiak RS, Hajnal JV, Woods RP, Mazziotta JC, Shipp S, Zeki S (1993) Area V5 of the human brain: a combined study using PET and MRI. Cereb Cortex 3:79-94. 